\begin{tabular}{|c|c|c|c|}
\hline$\Omega$ & D & $\equiv$ & 라 \\
\hline Claire L. Shovlin ${ }^{1,2}$ & $\begin{array}{l}1 \text { NHLI Cardiovascular Sciences, } \\
\text { Imperial College, London, } \\
\text { UK. } 2 \text { Respiratory Medicine, } \\
\text { Hammersmith Hospital, Imperial } \\
\text { College Healthcare NHS Trust, } \\
\text { London UK. }\end{array}$ & $\begin{array}{l}\text { Claire L. Shovlin, NHLI } \\
\text { Cardiovascular Sciences, } \\
\text { Imperial Centre for } \\
\text { Translational and Experimental } \\
\text { Medicine, Imperial College } \\
\text { London, Hammersmith } \\
\text { Campus, Du Cane Road, } \\
\text { London, W12 oNN, UK }\end{array}$ & c.shovlin@imperial.ac.uk \\
\hline
\end{tabular}

\title{
Curable hypoxia in an octogenarian with an undiagnosed inherited condition: a case commentary
}

\section{Introduction}

The accompanying case is an excellent illustration of why pulmonary arteriovenous malformations (PAVMs) have been considered very rare. Patients with PAVMs are generally asymptomatic and may be highly athletic, even in the presence of profound hypoxaemia due to right-to-left shunting through PAVMs. Data in papers published this year help explain why patients are so rarely symptomatic [1-3]; why some become symptomatic [1, 2]; and why, irrespective of respiratory symptoms, all PAVMs should be considered for treatment $[4,5]$.

\section{Why did this patient not have symptoms until after retirement?}

PAVM development is usually complete by the end of puberty, and the octogenarian in this case would have spent decades undiagnosed with "silent" PAVMs and hypoxaemia. To understand how this could be the case, it is important to realise that, although this patient had low blood oxygen levels, his tissues were not more "hypoxic" than normal. Instead, as recently emphasised [1-3], PAVM patients use multiple mechanisms to maintain oxygen delivery.
First, the relevant term for oxygen transport is not the arterial oxygen tension $\left(\mathrm{PaO}_{2}\right)$ or arterial oxygen saturation $\left(\mathrm{SaO}_{2}\right)$, but the arterial oxygen content $\left(\mathrm{CaO}_{2}\right)$ which also depends upon the concentration of haemoglobin [6]. Hypoxaemic PAVM patients generally utilise polycythaemic responses so that, irrespective of the $\mathrm{SaO}_{2}$, $\mathrm{CaO}_{2}$ on standing is preserved at just under 18 $\mathrm{mL} \cdot \mathrm{dL}-1[1]$.

Secondly, the overall transport of oxygen to the tissues also depends on the volume of blood reaching the tissues in any given period (cardiac output) [6]. Cardiac output is the product of the heart rate and stroke volume, and both are increased in PAVM patients to compensate for hypoxaemia [2, 3]. For example, in the 165 PAVM patients reported recently, orthodeoxia (a fall in blood oxygen on standing) was not accompanied by dyspnoea/platypnoea [4]. Instead, an exuberant orthostatic tachycardia was evident [4]. In our experience, if this is not possible, for example due to cardiac disease or beta-blockers, patients report more general symptoms including dizziness and the need to sit down [4].

Right-to-left shunts also impairs $\mathrm{CO}_{2}$ clearance, and patients may require exuberant ventilatory responses [2]. Surprisingly, although this appears to limit exercise tolerance [2], the extra ventilatory effort does not seem to be "sensed" by most PAVM patients as dyspnoea.[2].
Statement of Interest None declared. 
Thus, as for most PAVM patients with sizable right-to-left shunts, for many years, the patient was able to tolerate the resultant impaired gas exchange both at rest and on exercise.

\section{Why did this patient become breathless?}

It is possible that the patient's symptoms post-retirement resulted from deterioration in his PAVM/shunt, but this is quite unlikely. In our experience, for adult patients with $\mathrm{SaO}_{2}$ $>80 \%$, dyspnoea is usually only reported if there is an additional factor leading to inability to tolerate the right-to-left shunt; most commonly the onset of iron deficiency and/ or development of incidental cardiorespiratory pathologies.

As for most published cases, this patient's PAVM was due to hereditary haemorrhagic telangiectasia (HHT) [7], an inherited condition characterised by nosebleeds (which commonly lead to iron deficiency anaemia [8]), mucocutaneous telangiectasia and visceral arteriovenous malformations. Since hypoxaemic PAVM patients need to have a supranormal haemoglobin to maintain $\mathrm{CaO}_{2}$ [1], iron deficiency can be associated with symptoms even when haemoglobin values are in the normal range. In the current case, the patient reported frequent nosebleeds over a number of years, and dietary iron intake may not have always been sufficient for his needs [ 1 , 8]. Serial haematinics, iron indices and $\mathrm{SaO}_{2}$, standing were not provided, but iron deficiency and an impaired polycythaemic response [1] may have accounted for a component of his earlier symptomatic state.

The patient had concurrent airflow obstruction of moderate severity, and described both dyspnoea and wheeze. It is, therefore, highly relevant that in our consecutive series of 165 PAVM patients, dyspnoea was more likely to be reported if the patient had concurrent cardiorespiratory disease such as chronic obstructive pulmonary disease or asthma [4]. It is not possible to state whether his presentation primarily reflected: his airflow obstruction; the consequences of airflow obstruction making him less able to achieve the additional ventilatory and circulatory demands for shunt compensation; or a combination of both. However, exclusion and/or early optimisation of concurrent airflow obstruction are now a major component of management of PAVM patients in our service.

The important message for clinicians identifying PAVM-induced hypoxaemia in a patient reporting dyspnoea is that the PAVMs/ hypoxaemia are probably not the cause of the presentation, and alternative causes should be sought. That said there are a small number of individuals who seem less able to tolerate significant right-to-left shunting, the reasons for which are unclear.

\section{Treatment considerations}

All patients with PAVMs of a size amenable to embolisation should be considered for this generally safe and effective treatment, even if they have been asymptomatic for many decades.

In the presented case, the patient's exercise tolerance improved after treatment, but generally, predictions of symptomatic improvement should be made with caution. Just as PAVMs/right-to-left shunts are rarely the cause of dyspnoea in PAVM patients, patients cannot be guaranteed that any dyspnoea will improve, even if $\mathrm{PaO}_{2} / \mathrm{SaO}_{2}$ improves. Symptomatic improvements are more likely for PAVM patients with concurrent cardiorespiratory disease [3], particularly if associated with low serum albumin or higher pulmonary artery pressure [1]. Similarly, while PAVMs may be the cause of major haemoptysis/haemothorax, these are relatively rare symptoms unless the patient is pregnant or has a systemic arterial supply to PAVM sacs. In HHT patients, haemoptysis may reflect concurrent HHT, nasopharyngeal or endobronchial telangiectasia and, thus, persist after PAVM embolisation.

The main reason for treating PAVMs has always been, and remains, the prevention of major neurological complications due to paradoxical emboli, particularly ischaemic stroke and brain abscess [4, 5]. Both can be prevented by PAVM embolisation [9] as described elsewhere [10]. The case history does not report a clinical event, but more than $50 \%$ of PAVM patients displayed evidence of old cerebral infarcts using twentieth century scanning methods [11].

For a minority of patients presenting with PAVMs, embolisation will completely occlude all PAVMs. This is more likely for patients with single, relatively simple PAVMs, which are less likely to be related to underlying $\mathrm{HHT}$. 
However, the majority of patients, even after maximal embolisation, are left with residual PAVMs that are technically too small to treat. There is now good evidence that very low grade shunts (below the limit of computed tomography detection) are associated with lower neurological risks in the PAVM/HHT population [12]. Nevertheless, attention to medical and lifestyle manoeuvres to reduce the risk of stroke/abscess is likely to be required for the majority of PAVM patients [4, 10], even after embolisation.

\section{Family screening}

As discussed in the presented case, evaluation of other aspects of $\mathrm{HHT}$, including family screening, is also important. The latest data suggest that the proportion of PAVM patients with underlying HHT will be less than previously estimated, due to the rise in detection of PAVMs by incidental CT scans (current prevalence estimates; PAVMs $\sim 1$ in 2600 [13], compared with accepted figures of 1 in $5000-8000$ for HHT). Since HHT is often difficult to diagnose and the risks of silent PAVMs so substantial, [4, 9, 14] we err on the side of caution and tend to recommend PAVM screening after puberty for the children of PAVM patients, even if there is no clear evidence of $\mathrm{HHT}$ in the proband or wider family.
A normal chest radiograph cannot rule out the presence of small PAVMs which may nevertheless cause neurological sequelae [4, 9]. The question of whether to use contrast echocardiography (CE) as a first-line screening tool, or proceed directly to a CT scan, is debated. CE was recommended by the HHT international guidelines committee [15], but for the reasons discussed in detail elsewhere $[4,10]$, since 2002 we have generally used CT scans for a first-line screen: Completely negative CE studies are not often found in HHT patients (so most require a CT scan for further evaluation) [16], and positive CE studies have a low positive predictive value for CT-detectable PAVMs, particularly if shunting is not rigorously graded.

Information about other aspects of HHT is also important. The $1 \%$ risk of maternal death in pregnancy [14], the frequency of cerebral AVMs that may need neither treatment [17] nor detection, and the familial nature of $\mathrm{HHT}$, are just three of the highly challenging discussions that should take place. In our experience, awareness of the surprisingly good exercise capacity [1, 2], life expectancy [18] and apparently reduced rates of lung and other cancers in HHT [19], help place more difficult issues in perspective for individuals with HHT.

Dr Shovlin runs a UK national clinic for people with HHT and PAVMs.

\section{References}

1. Santhirapala V, Williams LC, Tighe HC, et al. Arterial oxygen content is precisely maintained by graded erythrocytotic responses in settings of high/normal serum iron levels, and predicts exercise capacity: an observational study of hypoxaemic patients with pulmonary arteriovenous malformations. PLoS One 2014; 9: e90777.

2. Howard LSGE, Santhirapala V, Murphy K, et al. Cardiopulmonary exercise testing demonstrates maintenance of exercise capacity in hypoxemic patients with pulmonary arteriovenous malformations. Chest 2014 [In press DOI: 10.1378/chest.13-2988].

3. Santhirapala V, Chamali B, McKernan $\mathrm{H}$, et al. Orthodeoxia and postural orthostatic tachycardia in patients with pulmonary arteriovenous malformations: a prospective 8-year series. Thorax 2014 [In press DOI: 10.1136/thoraxjnl-2014-205289].

4. Shovlin CL, Chamali B, Santhirapala V, et al. Ischaemic strokes in patients with pulmonary arteriovenous malformations and hereditary hemorrhagic telangiectasia: associations with iron deficiency and platelets. PLoS One 2014; 9: e88812.

5. Kjeldsen AD, Tørring PM, Nissen $\mathrm{H}$, et al. Cerebral abscesses among Danish patients with hereditary haemorrhagic telangiectasia. Acta Neurol Scand 2014 129: 192-197.

6. Pittman RN. Chapter 4 oxygen transport. In:
Regulation of Tissue Oxygenation. San Rafael, Morgan \& Claypool Life Sciences, 2011.

7. Shovlin CL. Hereditary haemorrhagic telangiectasia: pathophysiology, diagnosis and treatment. Blood Rev 2010; 24: 203-219.

8. Finnamore H, Le Couteur J, Hickson $M$, et al. Hemorrhage-adjusted iron requirements, hematinics and hepcidin define hereditary hemorrhagic telangiectasia as a model of hemorrhagic iron deficiency. PLoS One 2013; 8: e76516.

9. Shovlin CL, Jackson JE, Bamford KB, et al. Primary determinants of ischaemic stroke/brain abscess risks are independent of severity of pulmonary arteriovenous malformations in hereditary haemorrhagic telangiectasia. Thorax 2008; 63: 259-266.

10. Shovlin CL, Wilmshurst P, Jackson JE. Pulmonary arteriovenous malformations and other pulmonary aspects of HHT. In: Cordier J-F, ed. Orphan Lung Diseases. Eur Respir Monogr 2011; 54: 218-245.

11. Moussouttas M, Fayad P, Rosenblatt M, et al. Pulmonary arteriovenous malformations: cerebral ischemia and neurologic manifestations. Neurology 2000; 55: 959-964.

12. Velthuis S, Buscarini E, van Gent MW, et al. Grade of pulmonary right-to-left shunt on contrast echocardiography and cerebral complications: a striking association. Chest 2013; 144: 542-548.

13. Nakayama M, Nawa T, Chonan T, et al. Prevalence of 
pulmonary arteriovenous malformations as estimated by low-dose thoracic CT screening. Intern Med 2012; 51 : 1677-1681.

14. Shovlin CL, Sodhi V, McCarthy A, et al. Estimates of maternal risks of pregnancy for women with hereditary haemorrhagic telangiectasia (Osler-Weber-Rendu syndrome): suggested approach for obstetric services. BJOG 2008; 115: 1108-1115.

15. Faughnan ME, Palda VA, Garcia-Tsao G, et al. International guidelines for the diagnosis and management of hereditary haemorrhagic telangiectasia. J Med Genet 2011; 48: 73-87.

16. van Gent MW, Post MC, Snijder RJ, et al. Real prevalence of pulmonary right-to-left shunt according to genotype in patients with hereditary hemorrhagic telangiectasia: a transthoracic contrast echocardiography study. Chest 2010; 138: 833-839.

17. Mohr JP, Parides MK, Stapf C, et al . Medical management with or without interventional therapy for unruptured brain arteriovenous malformations (ARUBA): a multicentre, non-blinded, randomised trial. Lancet 2014; 383: 614-621.

18. Kjeldsen AD, Vase P, Green A. Hereditary haemorrhagic telangiectasia: a population-based study of prevalence and mortality in Danish patients. J Intern Med 1999; 245: 31-39.

19. Hosman AE, Devlin HL, Silva BM, et al. Specific cancer rates may differ in patients with hereditary haemorrhagic telangiectasia compared to controls. Orphanet J Rare Dis 2013; 8: 195. 